\title{
Overexpressing microRNA-150 attenuates hypoxia-induced human cardiomyocyte cell apoptosis by targeting glucose-regulated protein-94
}

\author{
JIAN-LIN MA ${ }^{1}$, WEN-LING GUO ${ }^{2}$ and XUE-MEI CHEN ${ }^{2}$ \\ Departments of ${ }^{1}$ Emergency and ${ }^{2}$ Obstetrics, Binzhou City Central Hospital, Binzhou, Shandong 251700, P.R. China
}

Received October 13, 2016; Accepted June 13, 2017

DOI: $10.3892 / \mathrm{mmr} .2018 .8375$

\begin{abstract}
MicroRNA (miR)-150 has been demonstrated to protect the heart from ischemic injury. However, the protective effect of miR-150 in hypoxia-injured cardiomyocytes remains unclear. The present study aimed to investigate the target gene of miR-150 and the underlying molecular mechanisms of miR-150 in hypoxia-induced cardiomyocyte apoptosis. Using the hypoxia model of human cardiomyocytes (HCMs) in vitro, it was demonstrated that miR-150 was markedly inhibited in HCMs after hypoxia treatment. Overexpressing miR-150 significantly decreased hypoxia-induced HCM death and apoptosis. In addition, GRP94 was revealed to be a direct target of miR-150. Additionally, GRP94 was demonstrated to be involved in hypoxia-induced HCM apoptosis, and the protein expression levels of GRP94 were increased in HCMs in the presence of hypoxia. These findings demonstrated that miR-150 is involved in hypoxia-mediated gene regulation and apoptosis in HCMs. Furthermore, GRP94 knockout increased the cell viability of hypoxia-impaired HCMs with miR-150 mimic or miR-150 inhibitor transfection. In conclusion, miR-150 may serve a protective role in cardiomyocyte hypoxia injury, and the underlying mechanism was mediated, at least partially, by inhibiting GRP94 expression. These findings may provide a novel insight for the therapy of hypoxia-induced myocardial I/R injury.
\end{abstract}

\section{Introduction}

The cardiovascular system provides blood flow to tissues, which delivers oxygen and nutrients to maintain normal metabolism for cells (1). Restriction of cardiac blood flow results in myocardial ischemia, which can trigger ischemia/reperfusion (I/R) injury, such as myocardial infarction

Correspondence to: Dr Jian-Lin Ma, Department of Emergency, Binzhou City Central Hospital, 108 South Huancheng Road, Binzhou, Shandong 251700, P.R. China

E-mail: m_lmam@163.com

Key words: microRNA-150, hypoxia, cardiomyocytes, glucoseregulated protein-94 and hemorrhage shock (2). The decrease in myocardial oxygen tension and rise in carbon dioxide tension contribute to the initiation of cell death by increasing generation of reactive oxygen species (ROS), disruption of calcium homeostasis, depletion of glycogen stores, mitochondrial injury and oxidative damage to DNA (3-5). The apoptosis of cardiomyocytes serves a vital role in the development of various biological and pathological processes, including ischemic injuries (6). Therefore, maintenance of normal myocardial cell function is of great significance for the treatment of myocardial ischemic injuries.

MicroRNAs (miRs) are a class of small non-coding RNA (18-25 nucleotides) molecules that regulate the translation of messenger RNAs (mRNAs) by binding to the 3'-untranslated regions (3'-UTR) of target mRNAs (7). Multiple studies have demonstrated that the pathogenic change in various tissues of humans has been associated with abnormal miR expression $(8,9)$. Notably, miRs have provided novel insights into hypoxia-induced myocardial cell dysfunction (10). It has been reported that $\mathrm{miR}-7 \mathrm{a} / \mathrm{b}$ attenuates post-myocardial infarction remodeling and protects $\mathrm{H} 9 \mathrm{c} 2$ cardiomyoblast against hypoxia-induced apoptosis, involving specificity protein 1 and poly (ADP-ribose) polymerase-1 (11). miR-221 is involved in survival of hypoxia preconditioned ventricular myocytes through the DNA damage-inducible transcript 4 protein/mechanistic target of rapamycin complex 1 and tumor protein p53-inducible nuclear protein 1/p62 signaling pathways (12). miR-21 and miR-146a synergistically decrease apoptosis under ischemia/hypoxic conditions in cardiomyocytes (13). However, the role of miRNAs in regulating hypoxia-induced myocardial cell injury has not been completely clarified and requires further investigation. Previous studies indicated that miR-150 protects the heart from injury by inhibiting monocyte accumulation and cell death in a mouse model of acute myocardial infarction (14,15). However, the specific role of miR-150 in hypoxia-induced myocardial cell dysfunction remains unknown.

Glucose-regulated protein-94 (GRP94) is a most abundant glycoprotein in the endoplasmic reticulum (ER), and is involved in the maintenance of cell survival by protecting against stresses due to $\mathrm{Ca}^{2+}$ depletion from the ER $(16,17)$. A previous study demonstrated that GRP94 is expressed in the sarcoplasmic reticulum (SR) of adult rabbit cardiomyocytes (18). GRP94 is reported to protect cardiomyocytes from both 
ischemia and calcium overload (19). However, the underlying molecular mechanisms of GRP94 in hypoxia-induced myocardial cell dysfunction have not been previously addressed. The present study demonstrated that miR-150 could regulate GRP94 expression by targeting to the 3'-UTR of GRP94, suggesting that a post-translational mechanism may exist for regulating GRP94 expression by miR-150 in hypoxia-treated cardiomyocytes, which might provide a potential therapeutic treatment for I/R injury.

\section{Materials and methods}

Cell culture treatments. Human cardiomyocytes (HCMs) were commercially obtained from ScienCell Research Laboratories (Carlsbad, CA, USA) and were maintained in Dulbecco's modified Eagle's medium (Thermo Fisher Scientific, Inc. Waltham, MA, USA) supplemented with $10 \%$ fetal bovine serum (cat. no. C0225; Beyotime Institute of Biotechnology, Haimen, China) at $37^{\circ} \mathrm{C}$ with $5 \% \mathrm{CO}_{2}, 94 \% \mathrm{~N}_{2}$ and $1 \% \mathrm{O}_{2}$ for $3,6,12,24$ and $48 \mathrm{~h}$.

MTT assay. HCM viability was assessed using an MTT Cell Proliferation/Viability Assay kit (R\&D Systems, Inc., Minneapolis, MN, USA) according to the manufacturer's protocol. The purple formazan was dissolved in dimethyl sulfoxide and the optical density value was read at $490 \mathrm{~nm}$ on a microplate reader (MD SpectraMax M5; Molecular Devices, LLC, Sunnyvale, CA, USA).

Apoptosis assay by Annexin V/propidium iodide (PI) and terminal deoxynucleotidyl transferase-mediated dUTP nick end labeling (TUNEL) staining. HCMs were harvested and washed with PBS. The percentage of normal (non-apoptotic) and apoptotic cells was measured by double supravital staining with Annexin-V and PI, using an Annexin V-FITC Apoptosis Detection kit (Beyotime Institute of Biotechnology, Haimen, China). Flow cytometric analysis used a Cytomics FC500 flow cytometer with CXP software (version 5.0; Beckman Coulter, Inc., Brea, CA, USA). Additionally, hypoxia-induced apoptosis was assessed by TUNEL staining. The TUNEL assay was performed using a TUNEL Apoptosis kit (Beyotime Institute of Biotechnology) according to the manufacturer's protocol.

Measurement of lactate dehydrogenase ( $L D H)$ activity. The LDH activity in the culture medium was detected using an LDH assay kit (Nanjing Jiancheng Bioengineering Institute, Nanjing China) for the measurement of cell injury, according to the manufacturer's protocol.

Caspase-3 activity and cell apoptosis assay. HCMs lysates were prepared and incubated with an anti-caspase 3 antibody (cat.no. sc7272; 1:200; Santa Cruz Biotechnology, Inc., Dallas, TX, USA) at $37^{\circ} \mathrm{C}$ for $1 \mathrm{~h}$. Immunocomplexes were incubated with peptide substrate in assay buffer for $2 \mathrm{~h}$ at $37^{\circ} \mathrm{C}$. Release of p-nitroaniline was measured at $405 \mathrm{~nm}$ using an ELISA reader (MD SpectraMax M5) according to the manufacturer's protocol.

miR target prediction. The potential binding site between miR-124 and MCP-1 were obtained using online predict software (miRanda-mirSVR; http://www.microrna.org), miRDB (http://www.mirdb.org/miRDB/) and TargetScan (http://www.targetscan.org/).

Retrovirus package, transduction and transfection. Control short hairpin (sh)RNA and specific sh-RNAs targeting GRP94 (cat. no. sc-44304) were purchased from Santa Cruz Biotechnoogy, Inc. (Dallas, TX, USA), and the corresponding sequences were cloned into the pSIREN-RetroQ plasmid (Addgene, Inc.) for retrovirus production with HCMs.

Fortransfection,theFAMmodified2'-OMe-oligonucleotides were chemically synthesized and purified by high-performance liquid chromatography (Shanghai GenePharma, Co., Ltd., Shanghai, China). The miR-150 mimics were composed of RNA duplexes with the following sequence: 5'-UCUCCC AACCCUUGUACCAGUG-3'. The sequences of miR-150 inhibitor and scramble oligonucleotides were as follows: 5'-CACUGGUACAAGGGUUGGGAGA-3'; and 5'-CCGAAA CCUCGGUUGAUUGCGG-3'. Cells were transfected using Lipofectamine 2000 (Invitrogen; Thermo Fisher Scientific, Inc.) at a final concentration of $100 \mathrm{nM}$. At $24 \mathrm{~h}$ post-transfection, the culture medium was changed. After $48 \mathrm{~h}$, cells were harvested for analysis.

Reverse transcription-quantitative polymerase chain reaction (RT-qPCR). RNA extraction was performed using TRIzol reagent, according to the manufacturer's protocol (Invitrogen; Thermo Fisher Scientific, Inc.). Synthesis of cDNA was performed by RT reactions with $4 \mu \mathrm{g}$ total RNA using Moloney murine leukemia virus reverse transcriptase (Invitrogen; Thermo Fisher Scientific, Inc.) with oligo dT (15) primers (Fermentas; Thermo Fisher Scientific, Inc.) as described by the manufacturer. miR-150 level was quantified by the mirVana qRT-PCR miRNA detection kit (Ambion; Thermo Fisher Scientific, Inc.) in conjunction with qPCR with SYBR Green (Bio-Rad Laboratories, Inc., Hercules, CA, $\mathrm{USA} ; 95^{\circ} \mathrm{C}$ for $5 \mathrm{~min}$, followed by 35 cycles of $95^{\circ} \mathrm{C}$ for $15 \mathrm{sec}$, $60^{\circ} \mathrm{C}$ for $30 \mathrm{sec}$, and $72^{\circ} \mathrm{C}$ for $30 \mathrm{sec}$ ). After circle reaction, the quantitation cycle $(\mathrm{Cq})$ was determined and relative miR-150 level was calculated based on the $\mathrm{Cq}$ values and normalized to U6 level in each sample using the $2^{-\Delta \Delta C q}$ method (20). PCR was performed with the following primers: GRP94, forward 5'-AGCAAGACGTGTTCGATTC-3' and reverse 5'-CCTCAA TTTTGTCAAGGGTG-3'; GAPDH, forward 5'-GCACCG TCAAGCTGAGAAC-3' and reverse 5'-TGGTGAAGACGC CAGTGGA-3'.

Western blotting. HCMs were extracted in NP-40 buffer, followed by 5-10 min boiling and centrifugation (12,000 $\mathrm{x}$ g for $15 \mathrm{~min}$ at $4^{\circ} \mathrm{C}$ ) to obtain the supernatant. Samples containing $50 \mu \mathrm{g}$ protein were separated by $10 \%$ SDS-PAGE and transferred to nitrocellulose membranes (Bio-Rad Laboratories, Inc.). After blocking with 5\% (w/v) non-fat dry milk in TBS and $0.1 \%$ (w/v) Tween 20 (TBST), the membranes were incubated with an anti-GRP94 primary antibody (cat. no. sc-32249; 1:500; Santa Cruz Biotechnology, Inc.) at room temperature for $2 \mathrm{~h}$ and normalized to $\beta$-actin (cat. no. sc-130065; 1:2,000; Santa Cruz Biotechnology, Inc.) at room temperature for $2 \mathrm{~h}$. After three washes with TBST, the membranes were next incubated with a horseradish peroxidase-conjugated secondary antibody 
(cat. no. sc516102; 1:10,000; Santa Cruz Biotechnology, Inc.) at room temperature for $2 \mathrm{~h}$ and subsequently visualized with chemiluminescence (Thermo Fisher Scientific, Inc.). Signals were assessed using Quantity One ${ }^{\circledR}$ software (version 4.5; Bio Rad Laboratories, Inc.).

Statistical analysis. Data are presented mean \pm standard error for each group. All statistical analyses were performed by using PRISM version 6.0 (GraphPad Software, Inc., La Jolla, CA, USA). Inter-group differences were analyzed by one-way analysis of variance followed by Tukey's post hoc test to compare the group means. $\mathrm{P}<0.05$ was considered to indicate a statistically significant difference.

\section{Results}

Hypoxia induces HCM death. The effect of hypoxia-induced HCMs apoptosis was examined by the MTT assay and TUNEL assay. Compared with the control group, the cell viability was significantly decreased following exposure to hypoxia in a time-dependent manner (Fig. 1A). TUNEL analysis was performed, and the results demonstrated that the relative percentage of living cells after hypoxia treatment for 12 or $24 \mathrm{~h}$ markedly decreased compared with the control group (Fig. 1B). Furthermore, caspase-3 (Fig. 1C) and LDH (Fig. 1D) levels were increased in HCMs subjected to hypoxia for 12 or $24 \mathrm{~h}$, which indicated that HCMs suffered hypoxia-associated injuries.

Hypoxia inhibits miR-150 expression in HCMs. The levels of miR-150 was detected by RT-qPCR assay, and the results demonstrated that miR-150 expression levels in the hypoxia-treated group decreased by nearly 56,48 and $40 \%$ at 12,24 and $48 \mathrm{~h}$, respectively, vs. the control group (Fig. 2A). To validate whether miR-150 mimics or inhibitors stable expression in HCMs, miR-150 mimics or inhibitors was transfected into HCMs by adenovirus-mediated gene transfer. RT-qPCR confirmed the elevated level of miR-150 in HCMs transfected with miR-150 mimics and the reduced level of miR-150 in HCMs transfected with miR-150 inhibitors (Fig. 2B). As hypoxia treatment was already observed to markedly inhibit miR-150 expression in HCMs, to validate whether miR-150 regulates hypoxia-induced HCMs apoptosis, MTT assay and Annexin V/PI double-staining followed by flow cytometry analysis were performed. As presented in Fig. 2C, HCMs transfected with miR-150 mimics exhibited a significant increase in cell viability in the presence of hypoxia. However, miR-150 inhibitors markedly reinforced hypoxia-induced decrease in cell viability. Overexpression of miR-150 markedly reversed hypoxia-induced HCMs apoptosis, whereas miR-150 inhibitors significantly increased cell apoptosis in the presence of hypoxia (Fig. 2D and E).

GRP94 is a direct target of miR-150. To explore the molecular mechanism of miR-150 in hypoxia-induced HCMs apoptosis, bioinformatics analysis indicated that GRP94 is a potential target of miR-150 in mammals (Fig. 3A), which serves a critical role in ER stress-induced cardiomyocyte apoptosis and heart failure (21). To validate whether miR-150 regulates GRP94 directly through a putative binding site in HCMs, the
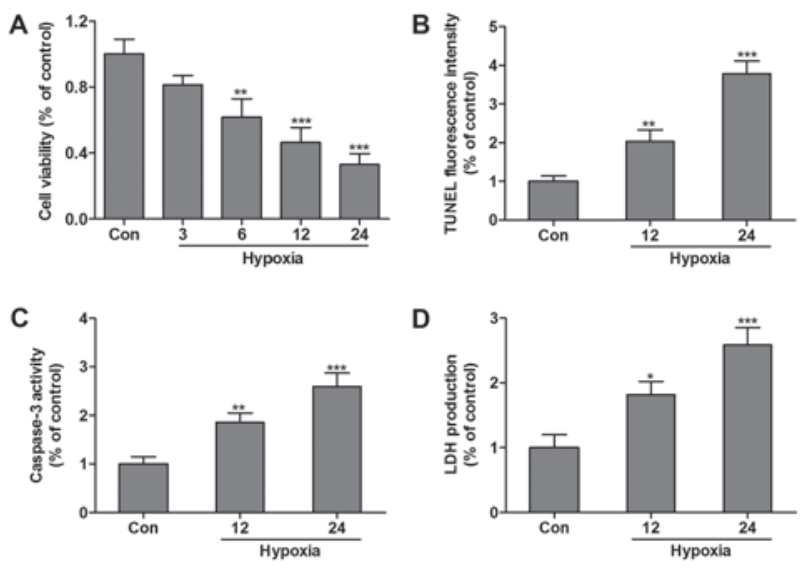

Figure 1. Hypoxia induces HCM death. (A) HCM proliferation was assessed by MTT assay in the present of hypoxia, and (B) cell apoptosis was measured by TUNEL analysis. (C) Caspase-3 and (D) LDH levels were increased during 0-24 h hypoxia treatment in HCMs. Data are expressed as the mean \pm standard error ( $\mathrm{n}=3$ /group). ${ }^{*} \mathrm{P}<0.05,{ }^{* *} \mathrm{P}<0.01,{ }^{* * *} \mathrm{P}<0.001$ vs. control group. TUNEL, terminal deoxynucleotidyl transferase-mediated dUTP nick end labeling; HCM, human cardiomyocyte; LDH, lactate dehydrogenase.
A
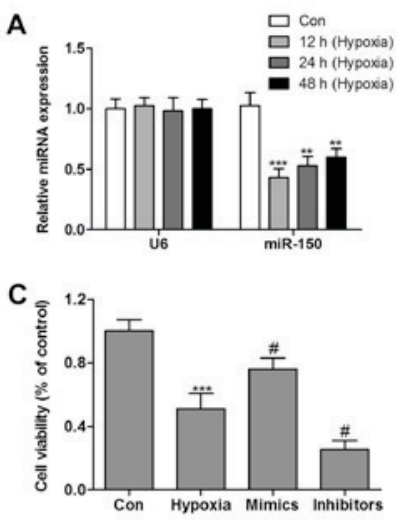

D

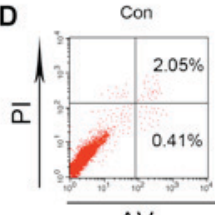

AV

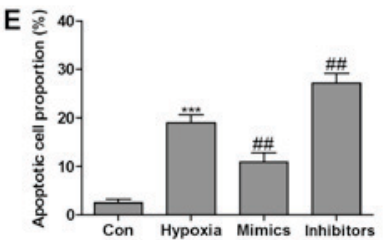

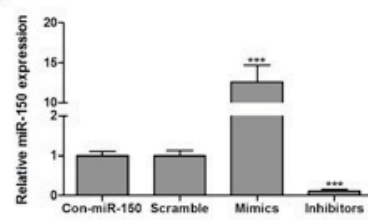

Figure 2. Hypoxia inhibits miR-150 expression in HCMs. (A) RT-qPCR analysis of miR-150 in HCMs after hypoxia treatment. (B) Detection of miR-150 expression in HCMs cells transfected with miR-150 mimics or miR-150 inhibitors by RT-qPCR. (C) MTT was performed to measure cell viability in HCMs transfected with the miR-150 mimics or inhibitors in the present of hypoxia. (D) Flow cytometry and (E) quantification of hypoxia-induced apoptosis in HCMs transfected with the miR-150 mimics or inhibitors. Data are expressed as the mean \pm standard error ( $\mathrm{n}=3$ /group). ${ }^{*} \mathrm{P}<0.05,{ }^{* * *} \mathrm{P}<0.01,{ }^{* * * *} \mathrm{P}<0.001$ vs control group; ${ }^{\#} \mathrm{P}<0.05,{ }^{\# \#} \mathrm{P}<0.01$ vs. hypoxia single treatment group. RT-qPCR, reverse transcription-quantitative polymerase chain reaction; HCM, human cardiomyocyte; Con, control; PI, propidium iodide; miR, microRNA.

GRP94 3'-UTR (wild-type or mutant) in the predicted miRNA binding site was cloned into the luciferase gene (pSiCHECK2). 

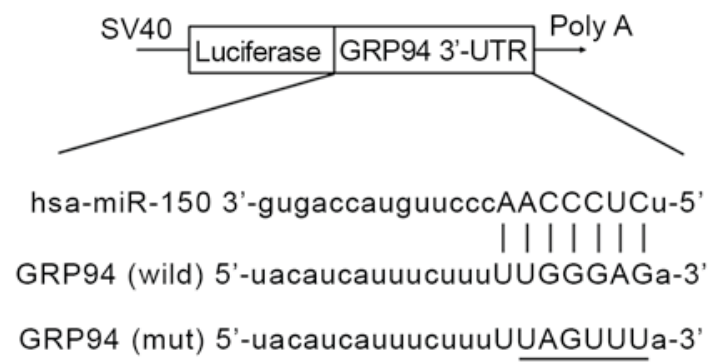

B

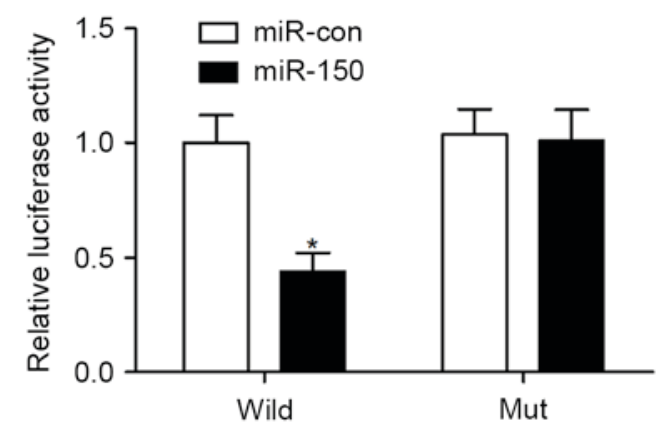

C
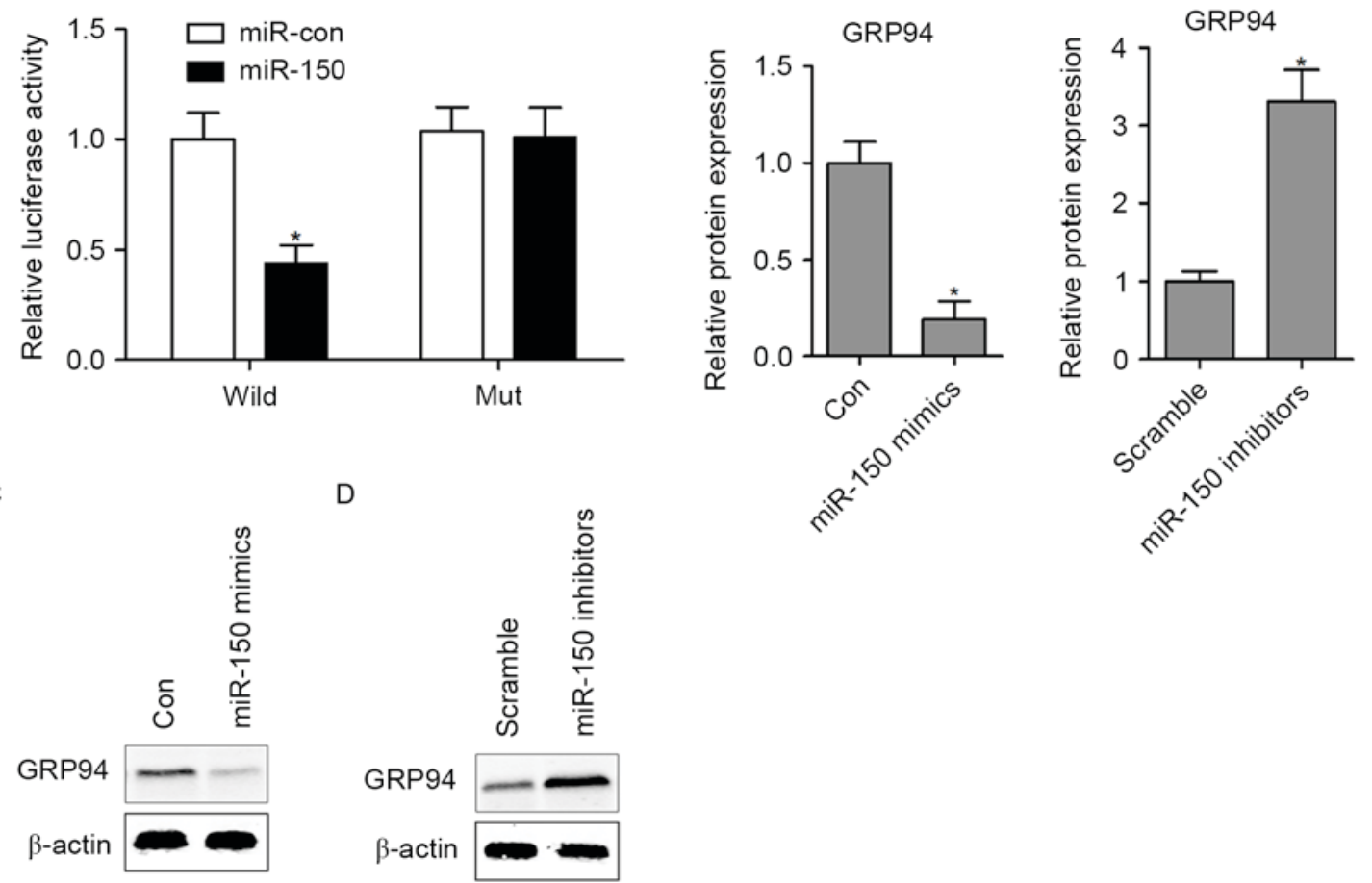

D

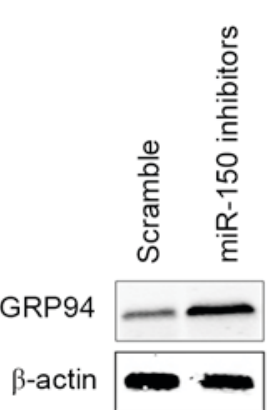

Figure 3. GRP94 is a direct target of miR-150. (A) The putative binding sites of miR-150 on the 3'-UTR of GRP94 is highlighted. (B) In a dual-luciferase reporter assay, HCMs were transfected with firefly luciferase reporter inserted with wild GRP94 3'-UTR or the reporter inserted with Mut 3'-UTR of GRP94, the relative luciferase activities were evaluated at $24 \mathrm{~h}$ after transfection. The protein expression of GRP94 transfected with (C) miR-150 mimics or (D) inhibitors, as assessed by western blotting. Data are expressed as the mean \pm standard error ( $\mathrm{n}=3$ /group). * $\mathrm{P}<0.05$ vs. control group. Mut, mutant; wild, wild-type; Con, control; UTR, untranslated region; GRP94, glucose-regulated protein 94; miR, microRNA.

Following cotransfection with the pSiCHECK2 vectors and miR-150 mimics or the control miRNA (miR-Con), the findings demonstrated that overexpressing miR-150 in HCMs significantly decreased luciferase activity in the wild-type GRP94 3'-UTR. However, following transfection of miR-150 mimics into GRP94 mutant HCMs, the luciferase activity did not exhibit a significant difference compared with the control group (Fig. 3B). To further assess whether miR-150 regulates GRP94 expression in HCMs, the expression of GRP94 was assessed by western blotting analysis in miR-150 mimic- or miR-150 inhibitor-transfected HCMs. The results indicated that overexpressing miR-150 markedly decreased the protein expression of GRP94 in HCMs (Fig. 3C). In contrast, inhibition of miR-150 in HCMs resulted in upregulation of GRP94 protein expression (Fig. 3D).

GRP94 is involved in the effect of miR-150 on hypoxia-induced HCMs apoptosis. The mRNA (Fig. 4A) and protein (Fig. 4B) expression levels of GRP94 were significantly increased in hypoxia-injured HCMs. To further understand the apoptosis mechanisms, sh-GRP94, miR-150 mimics or miR-150 inhibitors was co-transfected into hypoxia-injured HCMs. As presented in Fig. 4C, GRP94 knockout increased the cell viability of hypoxia-impaired HCMs with miR-150 mimics or miR-150 inhibitors transfection. Furthermore, GRP94 knockout could inhibit apoptosis and caspase-3 levels in hypoxia-impaired HCMs transfected with miR-150 mimics or miR-150 inhibitors (Fig. 4D and E).

\section{Discussion}

The results of the present study demonstrated that hypoxia directly induces HCM apoptosis, and the expression of miR-150 was markedly decreased in hypoxia-injured HCMs. Therefore, it was hypothesized that miR-150 may serve an important role in hypoxia-induced HCM apoptosis. Notably, further study demonstrated that overexpressing miR-150 significantly reversed hypoxia-induced HCM death. In contrast, miR-150 
A
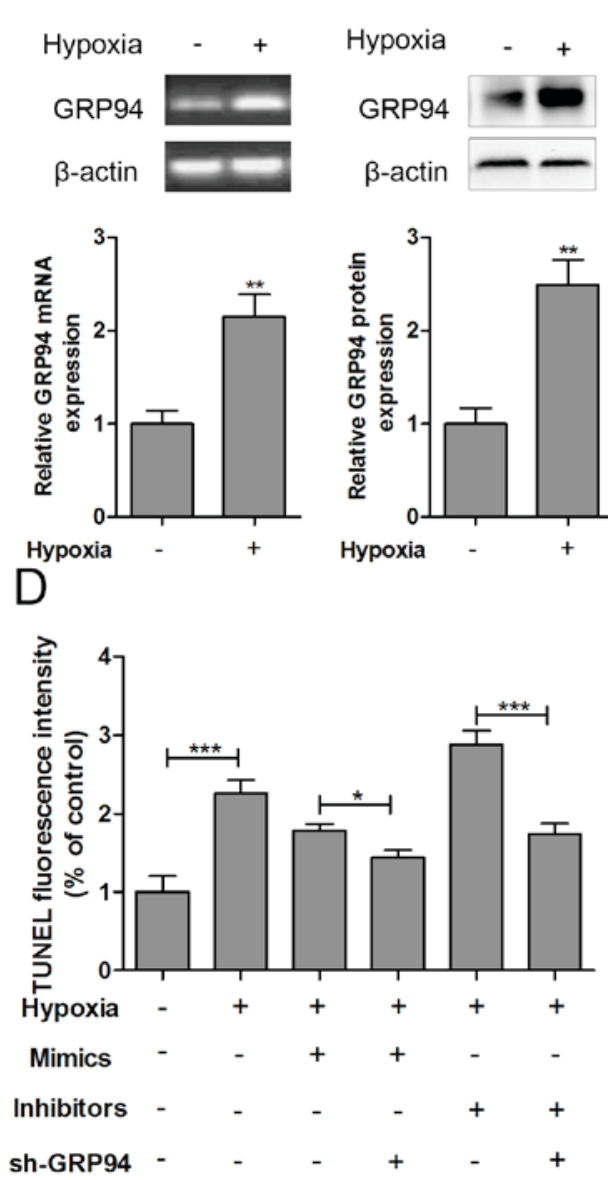

B
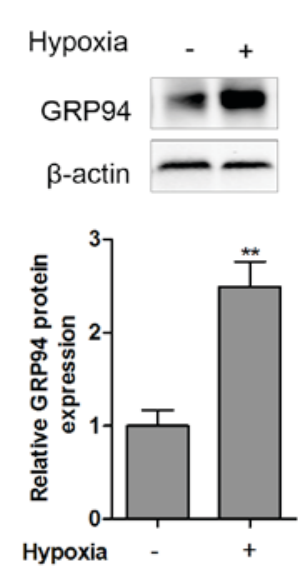

$\mathrm{E}$

C

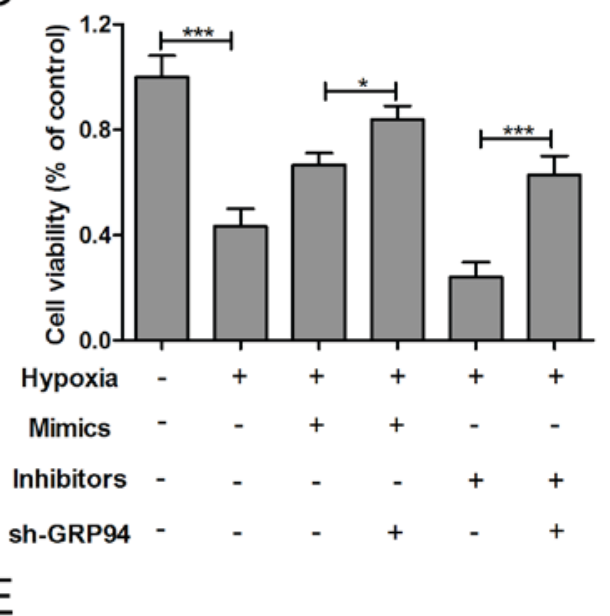

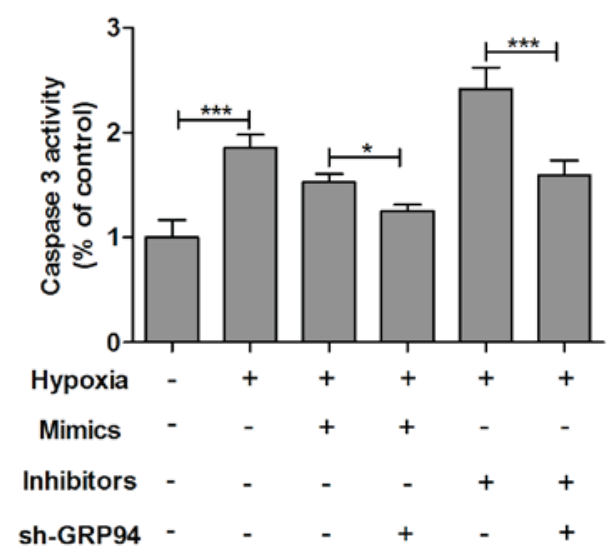

Figure 4. GRP94 is involved in the effect of miR-150 on hypoxia-induced HCM apoptosis. The (A) mRNA and (B) protein expression levels of GRP94 were measured in HCMs exposed to hypoxia for $24 \mathrm{~h}$. sh-GRP94, miR-150 mimics or miR-150 inhibitors was co-transfected into hypoxia-injured HCMs, and (C) cell viability, (D) TUNEL analysis and (E) caspase-3 levels were measured $12 \mathrm{~h}$ after treatment. Data are expressed as the mean \pm standard error ( $\mathrm{n}=3$ /group). ${ }^{*} \mathrm{P}<0.05,{ }^{* * *} \mathrm{P}<0.01,{ }^{* * *} \mathrm{P}<0.001$ vs. control group. HCM, human cardiomyocyte; sh, short hairpin; GRP94, glucose-regulated protein 94 ; miR, microRNA; TUNEL, terminal deoxynucleotidyl transferase-mediated dUTP nick end labeling; Con, control; wild, wild-type; mut, mutant.

inhibitors markedly reinforced a hypoxia-induced decrease in cell apoptosis. The molecular mechanism findings indicated that GRP94 as a direct target of miR-150 was involved in hypoxia-induced HCM apoptosis. These findings suggested that downregulation of GRP94 expression by miR-150 or GRP94 knockout protected against hypoxia-induced HCMs apoptosis.

miR-150 is strictly expressed in cardiomyocytes and cardiac fibroblasts, and serves a pivotal role of cardiomyocyte survival during cardiac injury $(14,22)$. Overexpressing miR-150 prevents high glucose-induced cardiomyocyte hypertrophy by targeting the transcriptional co-activator $\mathrm{p} 300$ (23). In contrast, miR-150 aggravates $\mathrm{H}_{2} \mathrm{O}_{2}$-induced cardiac myocyte injury by downregulating the c-myb gene (24). Furthermore, the expression of miR-150 in cardiac tissues is decreased in myocardial infarction and cardiac fibrosis induced by pressure overload (25). In addition, adenoviral overexpression of miR-150 causes a reduction in cardiomyocyte size (26). However, the potential roles of miR-150 have not been evaluated in hypoxia-induced HCM apoptosis. The present study further validated that miR-150 expression is decreased in hypoxia-injured HCMs. miR-150 deficiency reinforced hypoxia-induced HCM apoptosis. These findings suggested that downregulation of miR-150 at least partially promotes the progression of cardiovascular diseases.
A previous study verified that miR-150 expression is inhibited in hypoxia-induced hepatocytes (27). These research outputs suggested that miR-150 may be a novel anti-hypoxic miRNA, and reveal that miR-150 negatively regulates its target gene GRP94.

GRP94 as a molecular chaperone is involved in the maintenance of cell survival and has been demonstrated to serve important roles in ER stress-induced myocardial apoptosis (28). A previous study revealed GRP94 as the only SR calcium-binding protein that is increased during chronic atrial fibrillation (29). However, the upstream signals that regulate GRP94 expression in HCMs remain unclear. The present study demonstrated that miR-150 is capable of targeting the 3'-UTR of GRP94 to regulate hypoxia-induced HCMs apoptosis. The results also revealed that GRP94 knockout suppressed cell apoptosis of hypoxia-impaired HCMs transfected with miR-150 mimics or miR-150 inhibitors, suggesting that a post-transcriptional regulatory mechanism exists between GRP94 and miR-150 in the context of hypoxia-induced HCM apoptosis.

In conclusion, these results suggested that the expression of miR-150 in HCMs is downregulated following hypoxia treatment. Overexpressing miR-150 inhibited hypoxia-induced HCM apoptosis by downregulating GRP94 expression. 
These findings might provide novel insight for the therapy of hypoxia-induced myocardial I/R injury.

\section{References}

1. Loor G and Schumacker PT: Role of hypoxia-inducible factor in cell survival during myocardial ischemia-reperfusion. Cell Death Differ 15: 686-690, 2008.

2. Lin KH, Kuo WW, Jiang AZ, Pai P, Lin JY, Chen WK, Day CH, Shen CY, Padma VV and Huang CY: Tetramethylpyrazine ameliorated hypoxia-induced myocardial cell apoptosis via $\mathrm{HIF}-1 \alpha / \mathrm{JNK} / \mathrm{p} 38$ and IGFBP3/BNIP3 inhibition to upregulate PI3K/Akt survival signaling. Cell Physiol Biochem 36: 334-344, 2015.

3. Huang M, Nguyen P, Jia F, Hu S, Gong Y, de Almeida PE, Wang L, Nag D, Kay MA, Giaccia AJ, et al: Double knockdown of prolyl hydroxylase and factor-inhibiting hypoxia-inducible factor with nonviral minicircle gene therapy enhances stem cell mobilization and angiogenesis after myocardial infarction. Circulation 124 (11 Suppl): S46-S54, 2011.

4. Liang W, Guo J, Li J, Bai C and Dong Y: Downregulation of miR-122 attenuates hypoxia/reoxygenation (H/R)-induced myocardial cell apoptosis by upregulating GATA-4. Biochem Biophys Res Commun 478: 1416-1422, 2016.

5. Chang W, Song BW, Lim S, Song H, Shim CY, Cha MJ, Ahn DH, Jung YG, Lee DH, Chung JH, et al: Mesenchymal stem cells pretreated with delivered Hph-1-Hsp70 protein are protected from hypoxia-mediated cell death and rescue heart functions from myocardial injury. Stem Cells 27: 2283-2292, 2009.

6. Zhao Y, Xu L, Qiao Z, Gao L, Ding S, Ying X, Su Y, Lin N, He B and Pu J: YiXin-Shu, a ShengMai-San-based traditional Chinese medicine formula, attenuates myocardial ischemia/reperfusion injury by suppressing mitochondrial mediated apoptosis and upregulating liver-X-receptor $\alpha$. Sci Rep 6: 23025, 2016

7. Welten SM, Goossens EA, Quax PH and Nossent AY: The multifactorial nature of microRNAs in vascular remodelling. Cardiovasc Res 110: 6-22, 2016

8. Anadol E, Schierwagen R, Elfimova N, Tack K, Schwarze-Zander C, Eischeid H, Noetel A, Boesecke C, Jansen C, Dold L, et al: Circulating microRNAs as a marker for liver injury in human immunodeficiency virus patients. Hepatology 61 : 46-55, 2015.

9. Zhang WC, Chin TM, Yang H, Nga ME, Lunny DP, Lim EK, Sun LL, Pang YH, Leow YN, Malusay SR, et al: Tumour-initiating cell-specific miR-1246 and miR-1290 expression converge to promote non-small cell lung cancer progression. Nat Commun 7 : $11702,2016$.

10. Xu Y, Zhu W, Wang Z, Yuan W, Sun Y, Liu H and Du Z: Combinatorial microRNAs suppress hypoxia-induced cardiomyocytes apoptosis. Cell Physiol Biochem 37: 921-932, 2015

11. Li R, Geng HH, Xiao J, Qin XT, Wang F, Xing JH, Xia YF, Mao Y, Liang JW and Ji XP: miR-7a/b attenuates post-myocardial infarction remodeling and protects $\mathrm{H} 9 \mathrm{c} 2$ cardiomyoblast against hypoxia-induced apoptosis involving Sp1 and PARP-1. Sci Rep 6: 29082, 2016.

12. Chen Q, Zhou Y, Richards AM and Wang P: Up-regulation of miRNA-221 inhibits hypoxia/reoxygenation-induced autophagy through the DDIT4/mTORC1 and Tp53inp1/p62 pathways. Biochem Biophys Res Commun 474: 168-174, 2016.

13. Huang W, Tian SS, Hang PZ, Sun C, Guo J and Du ZM: Combination of microRNA-21 and microRNA-146a attenuates cardiac dysfunction and apoptosis during acute myocardial infarction in mice. Mol Ther Nucleic Acids 5: e296, 2016.

14. Tang Y, Wang Y, Park KM, Hu Q, Teoh JP, Broskova Z, Ranganathan P, Jayakumar C, Li J, Su H, et al: MicroRNA-150 protects the mouse heart from ischaemic injury by regulating cell death. Cardiovasc Res 106: 387-397, 2015.
15. Liu Z, Ye P, Wang S, Wu J, Sun Y, Zhang A, Ren L, Cheng C, Huang $X$ and Wang K: MicroRNA-150 protects the heart from injury by inhibiting monocyte accumulation in a mouse model of acute myocardial infarction. Circ Cardiovasc Genet 8: 11-20, 2015.

16. Vitadello M, Ausma J, Borgers M, Gambino A, Casarotto DC and Gorza L: Increased myocardial GRP94 amounts during sustained atrial fibrillation: A protective response? Circulation 103: 2201-2206, 2001

17. Little E and Lee AS: Generation of a mammalian cell line deficient in glucose-regulated protein stress induction through targeted ribozyme driven by a stress-inducible promoter. J Biol Chem 270: 9526-9534, 1995.

18. Vitadello M, Colpo P and Gorza L: Rabbit cardiac and skeletal myocytes differ in constitutive and inducible expression of the glucose-regulated protein GRP94. Biochem J 332: 351-359, 1998.

19. Vitadello M, Penzo D, Petronilli V, Michieli G, Gomirato S, Menabò R, Di Lisa F and Gorza L: Overexpression of the stress protein Grp94 reduces cardiomyocyte necrosis due to calcium overload and simulated ischemia. FASEB J 17: 923-925, 2003.

20. Livak KJ and Schmittgen TD: Analysis of relative gene expression data using real-time quantitative PCR and the 2(-Delta Delta C(T)) method. Methods 25: 402-408, 2001

21. Liu X, Kwak D, Lu Z, Xu X, Fassett J, Wang H, Wei Y, Cavener DR, Hu X, Hall J, et al: Endoplasmic reticulum stress sensor protein kinase R-like endoplasmic reticulum kinase (PERK) protects against pressure overload-induced heart failure and lung remodeling. Hypertension 64: 738-744, 2014

22. Deng P, Chen L, Liu Z, Ye P, Wang S, Wu J, Yao Y, Sun Y, Huang X, Ren L, et al: MicroRNA-150 inhibits the activation of cardiac fibroblasts by regulating c-Myb. Cell Physiol Biochem 38: 2103-2122, 2016.

23. Duan Y, Zhou B, Su H, Liu Y and Du C: miR-150 regulates high glucose-induced cardiomyocyte hypertrophy by targeting the transcriptional co-activator p300. Exp Cell Res 319: 173-184, 2013.

24. Li X, Kong M, Jiang D, Qian J, Duan Q and Dong A: MicroRNA-150 aggravates $\mathrm{H} 2 \mathrm{O} 2$-induced cardiac myocyte injury by down-regulating c-myb gene. Acta Biochim Biophys Sin (Shanghai) 45: 734-741, 2013

25. Devaux Y, Vausort M, McCann GP, Zangrando J, Kelly D, Razvi N, Zhang L, Ng LL, Wagner DR and Squire IB: MicroRNA-150: A novel marker of left ventricular remodeling after acute myocardial infarction. Circ Cardiovasc Genet 6: 290-298, 2013.

26. van Rooij E, Sutherland LB, Liu N, Williams AH, McAnally J, Gerard RD, Richardson JA and Olson EN: A signature pattern of stress-responsive microRNAs that can evoke cardiac hypertrophy and heart failure. Proc Natl Acad Sci USA 103: 18255-18260, 2006.

27. Yu ZY, Bai YN, Luo LX, Wu H and Zeng Y: Expression of microRNA-150 targeting vascular endothelial growth factor-A is downregulated under hypoxia during liver regeneration. Mol Med Rep 8: 287-293, 2013.

28. Shi ZY, Liu Y, Dong L, Zhang B, Zhao M, Liu WX, Zhang X and Yin XH: Cortistatin improves cardiac function after acute myocardial infarction in rats by suppressing myocardial apoptosis and endoplasmic reticulum stress. J Cardiovasc Pharmacol Ther: Apr 18, 2016 (Epub ahead of print).

29. Lai LP, Su MJ, Lin JL, Lin FY, Tsai CH, Chen YS, Huang SK, Tseng YZ and Lien WP: Down-regulation of L-type calcium channel and sarcoplasmic reticular $\mathrm{Ca}(2+)$-ATPase mRNA in human atrial fibrillation without significant change in the mRNA of ryanodine receptor, calsequestrin and phospholamban: An insight into the mechanism of atrial electrical remodeling. J Am Coll Cardiol 33: 1231-1237, 1999.

This work is licensed under a Creative Commons Attribution-NonCommercial-NoDerivatives 4.0 International (CC BY-NC-ND 4.0) License. 